\title{
Evaluation of the therapeutic efficacy of active specialistic medical dressings in the treatment of decubitus
}

\author{
Michał Senejko', Jarosław Pasek², Sebastian Szajkowski³, Grzegorz Cieślar', Aleksander Sieroń \\ 'Department of Internal Medicine, Angiology and Physical Medicine, Faculty of Medical Sciences in Zabrze, \\ Medical University of Silesia in Katowice, Poland \\ ${ }^{2}$ Faculty of Health Sciences, University of Jan Długosz, Częstochowa, Poland \\ ${ }^{3}$ Medical University of Mazovia, Warsaw, Poland
}

Adv Dermatol Allergol 2021; XXXVIII (1): 75-79

DOI: https://doi.org/10.5114/ada.2021.104282

\begin{abstract}
Introduction: Treatment of decubitus ulcers is a grave medical problem. In many cases, it is difficult to cure a pressure ulcer, especially when it is deep and extensive, and prognosis is usually unfavourable. Treatment of decubitus ulcers requires new specialist dressings, which play an important role in the healing process.

Aim: To evaluate therapeutic efficacy of active specialist medical dressings in the treatment of decubitus.

Material and methods: Research involved 40 patients - 18 (45\%) women and 22 (55\%) men, suffering from decubitus ulcers of different size and depth, localized in the sacral region, lasting from 1.5 to 30 months. Patients were randomly assigned to two research groups (20 people each), were treated for 4 weeks with 2 different specialist dressings. ATRAUMAN Ag, which contains silver ions, was used in the first group, while paraffin gauze of BACTIGRAS type was used in the second group. An assessment of pressure ulcers' healing progress was done with a planimetric method, which evaluates the wound surface area.

Results: The analysis results showed a significant statistical decrease in an average decubitus ulcer surface area in both research groups: in the first group by $60.2 \%(p=0.001)$, and in the second group by $32.95 \%(p<0.001)$, which speaks in favour of dressings with silver ions as having better therapeutic effectiveness.

Conclusions: Using specialist dressings results in a significant decrease in the decubitus ulcer surface area, depending on the type of dressing and active substances contained within, while silver ions support curative effectiveness of the dressing used.
\end{abstract}

Key words: decubitus, treatment, specialist local dressings, computerized planimetry.

\section{Introduction}

Decubitus ulcers are chronic wounds developed from a long-lasting constriction, friction or shearing force, which leads to vascular occlusion and trophic lesions of the skin. This state can affect all layers of epidermis. People who stay in a lying or sitting position for a longer period of time are at risk of developing decubitus ulcers. Areas most prone to develop pressure ulcers are areas in which the layer of soft tissue separating the bone from the base is thin (trochanters, sacrum, ischial tuberosity, elbow joints and heels) [1, 2].

The pathophysiological mechanism of developing pressure ulcers is conditioned by many factors. The most prominent factor is pressure exceeding $20 \mathrm{~mm} \mathrm{Hg}$ exerted on the soft tissues depriving them of oxygen supply and nutrients due to vascular occlusion; friction caused by incorrect positions and lateral shear forces causing pathological movements of tissues against each other with recurrent rupture of capillary vessels, and disturbed microcirculation. As a result, hypoxic tissues deteriorate $[3,4]$.

An assessment of the medical condition based on a medical history, physical examination and definition of patient's treatment needs in regard to a non-healing decubitus should be performed as the first stage in treating patients with pressure ulcers. Unfortunately, it is

Address for correspondence: Jarosław Pasek PhD, Department of Internal Medicine, Angiology and Physical Medicine of Medical University of Silesia, 15 Stefana Batorego St, 41-902 Bytom, Poland, phone: +48 (32) 786-16-30, fax: + 48 (32) 786-16-30, e-mail: jarus_tomus@o2.pl Received: 13.04.2019, accepted: 15.07.2019. 
a complex process and it should be tailored individually to each patient.

The Torrance pressure sores grading system, of five grades, is used to assess the size of decubitus. In grade 1 pressure ulcer, there is a blanchable redness of the skin in which microcirculation is not yet damaged, therefore pressure posed by a finger causes blanching of the redness. In grade 2 pressure ulcer, there is a non-blanchable redness caused by the damaged microcirculation; skin damage on the surface, blisters and pain may also appear. In grade 3 pressure ulcer, there is a damage of all layers of the skin stretching to the subcutaneous tissue, lips of the wound are well-separated, surrounded by swelling and erythema, while the wound bed is filled with red granulation tissue or yellow masses of disintegrating tissue. In grade 4 pressure ulcer, damage covers the skin and subcutaneous tissue, lips of the wound are usually well-separated and the wound bed may be covered with eschar. In grade 5 pressure ulcer, necrosis also covers fascia and muscles, sometimes joints and bones; there are cavities and "pockets" which are joined together and the wound is filled with black and brown masses of disintegrating tissue.

Apart from pharmacological treatment and adherence to the rules of pressure ulcer prevention, localized specialist dressings can be used as an active supplement to the treatment. According to the CEAP recommendations, wounds are treated in the moist environment which allows for more intensive skin regeneration. The dressings include hydrocolloid, hydrogel and hydrofiber dressings as well as alginates and foam dressings. Optimal dressing should have physicochemical properties, which maintain the moist environment in the wound bed, cannot adhere to the surface of the wound, have sorption properties, should prevent recurring infections and be easy to remove. Currently, ionic silver is very often used as a material for specialist dressings as it has documented strong bactericidal effects [5-7].

\section{Aim}

The aim of the study was to compare the efficacy of specialist dressing ATRAUMAN Ag containing silver ions and classical dressing BACTIGRAS composed of sterile paraffin gauze in patients with pressure ulcers, basing on the planimetric assessment of the ulcer surface area.

\section{Material and methods}

Forty patients (18 women and 22 men) aged 64-80, residents of the Nursing Home in Olkusz, who were diagnosed with grade 1 and 2 pressure ulcers according to the Torrance classification, took part in the research. After a medical history was taken and physical examination was done, patients were randomly assigned to two research groups (20 people each) which were treated with two dif- ferent types of dressings. Both groups of patients did not differ statistically in terms of age (which was 79.0 years in the first group and 79.9 in the second), sex and associated diseases. Applied dressings were characterized by small thickness, thus putting little pressure on the wound. These dressings have properties ensuring moist environment which supports faster healing. They did not require to be changed every day, and when they were changed they did not damage a new layer of granulation tissue. The only contraindication to use them for treatment was a confirmed patients' allergy to any active substance.

Treatment procedures for both groups of patients were established at the Department of Internal Diseases, Angiology and Physical Medicine of the Medical University of Silesia in Katowice. In the first group, basing on a scale to assess the risk of decubitus formation by Norton, apart from previous surgical debridement, a typical local pharmacological treatment (antiseptic agent Octenisept) and routine procedures of pressure ulcer prevention, the ATRAUMAN Ag dressings containing silver ions were used; whereas, in the second group, basing on a scale to assess the risk of decubitus formation by Norton, apart from previous surgical debridement, a typical local pharmacological treatment (antiseptic agent Octenisept) and routine procedures of pressure ulcer prevention, similar to the first group of patients, the BACTIGRAS sterile paraffin gauze was used. Every patient was treated for 4 weeks during which dressings were changed every 3 days.

Before and after the treatment, in order to verify the healing results obtained, the planimetric assessment of the wound surface area in both groups was performed with the help of a computer program created by Michat Senejko, MSc (Figure 1).

Planimetric assessment of lesions via computer program was performed manually. In the first stage, the doctor loaded the analysed picture from a digital photo of a pressure ulcer with a subsequent selection of lesions' contours. The program allows for selection of areas of lesions in two modes. In the first mode, an analyst moves a mouse cursor continuously along a contour of the target area. After double-clicking the mouse cursor, the drawn contour is automatically closed, creating a closed curve. In the second mode, a selected contour is defined by indicating a set of points of a lesion's contour. In this mode, the program automatically connects each new point with previous points, as a result of which one gets a closed contour.

When the selection stage is done, the program automatically calculates the surface area within the previously defined contour. The surface area is represented in pixels or after an adequate calculation in square centimetres. In order to represent the surface area in square centimetres, it is necessary to calibrate the distance of the analysed body part from the camera lens. To do this, during shooting, the body part has to be marked with 


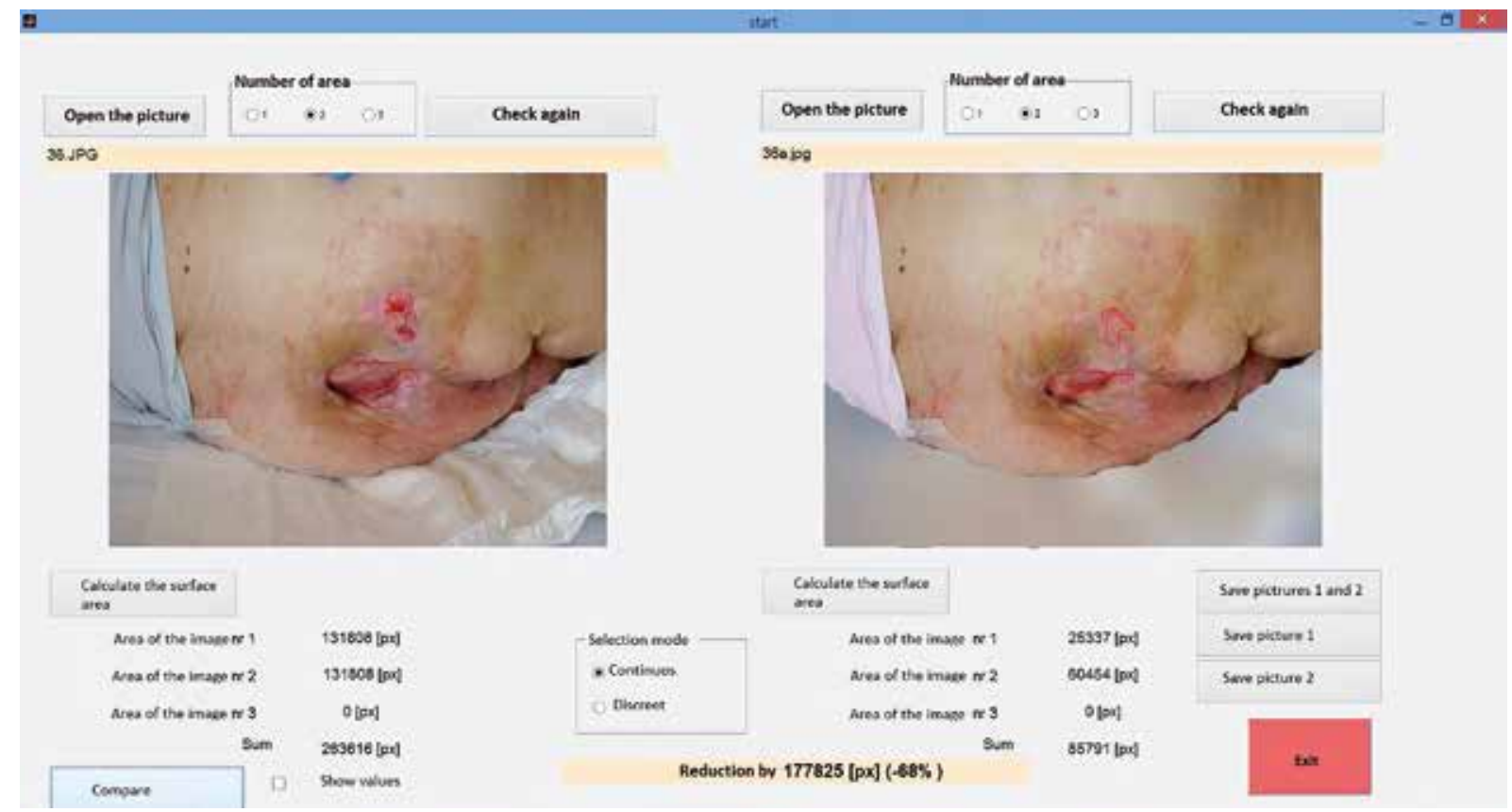

Figure 1. Planimetric assessment of the wound surface area before and after the treatment

a green square of size $1 \times 1 \mathrm{~cm}^{2}$. Thanks to this, the program can localize the aforementioned green square and calculate its size, and therefore by performing the scaling process it is able to assess the extent of the decubitus ulcer.

During treatment, all patients used a pressure-reducing mattress and their postural position was regularly changed.

\section{Statistical analysis}

Statistical analysis was done with Statistica PL 10.0 program. After a preliminary check, the nature of distribution of quantitative variables, statistical significance of differences of values of the analysed parameters before and after the treatment for and between both groups of patients were verified with the Wilcoxon signed-rank test and the Mann-Whitney $U$ test. The value of $p<0.05$ was assumed as statistical significance.

\section{Results}

A comparison of the wound surface areas for both groups, before and after the treatment (average value \pm standard deviation - SD) is shown in Figure 2.

Before the treatment, the average wound surface area in the first group of patients treated with ATRAUMAN Ag was $10.4 \pm 8.7 \mathrm{~cm}^{2}$ and was statistically significantly smaller $(p=0.013)$ in comparison to the second group of patients treated with BACTIGRAS, which was $17.0 \pm 11.3 \mathrm{~cm}^{2}$.
After the treatment the average wound surface area in the first group of patients treated with ATRAUMAN Ag was $4.4 \pm 4.1 \mathrm{~cm}^{2}$ and was statistically significantly smaller $(p<0.001)$ in comparison to the second group of patients treated with BACTIGRAS, which was $11.6 \pm 8.2 \mathrm{~cm}^{2}$ (Figure 3).

After the treatment, in both groups of patients, a statistically significant reduction in the surface wound area

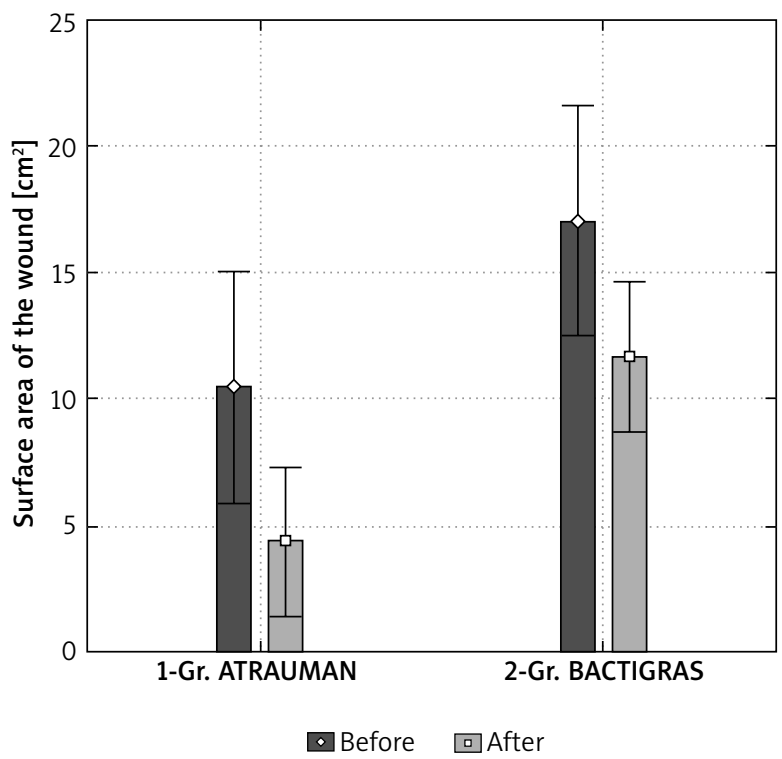

Figure 2. Comparison of the wound surface area in both groups of patients before and after the treatment (mean value \pm SD) 


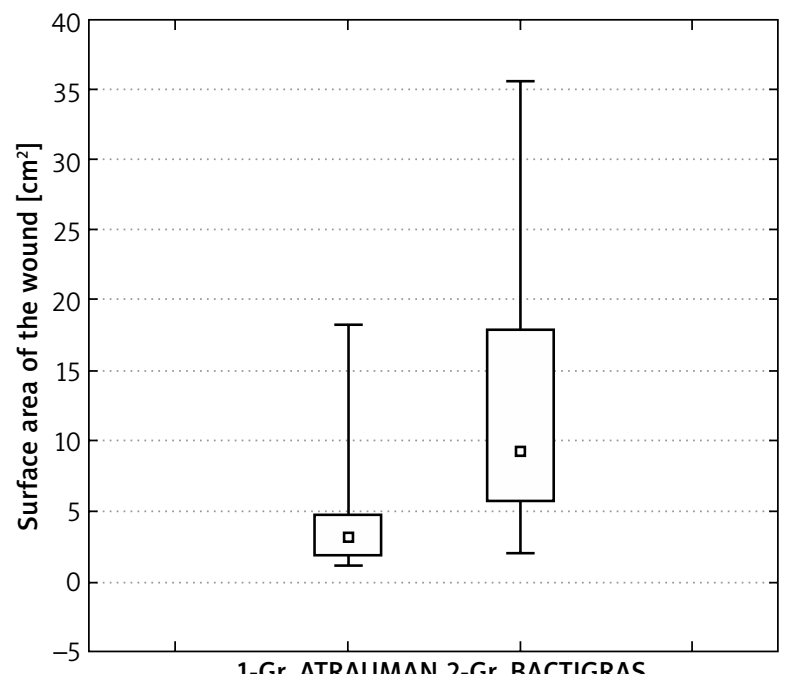

- Median

$\square 25-75 \%$

I Min.-max.

Figure 3. Comparison of the wound surface area in both groups of patients after the treatment

was observed in relation to output values, which in the first group treated with ATRAUMAN was $6.0 \mathrm{~cm}^{2}(p<$ 0.001 ), and in the second group treated with BACTIGRAS was $5.4 \mathrm{~cm}^{2}(p<0.001)$.

Percentage values of change of the surface wound area after the treatment in comparison to the output value for both groups is shown in Figure 4.

In the first group of patients treated with ATRAUMAN Ag, the reduction in the surface wound area was $60.2 \pm 12.0 \%$ on average; whereas in the second group of patients treated with BACTIGRAS, the reduction in the surface wound area was only $32.9 \pm 7.2 \%$ on average. The observed difference in percentage changes in the surface of the wound area between both groups of patients was statistically significant $(p=0.030)$.

\section{Discussion}

Knowledge about treatment of chronic wounds has been systematically broadened. It concerns also healing the decubitus ulcers which is a serious medical problem. Healing the pressure ulcers depends on their severity. Prognosis is usually unfavourable, especially in cases when the decubitus is deep and extensive [8-10]. In comprehensive punctate treatment it is important to use modern active dressings. The mechanism of action of such dressings allows for easier deterging of lesions in slough, reduces seepage and supports the granulation process. Thanks to their active ingredients, such as absorbent gels or sodium hyaluronate, they help to prepare optimal environmental conditions around lesions, which support a more intensive process of healing [11,

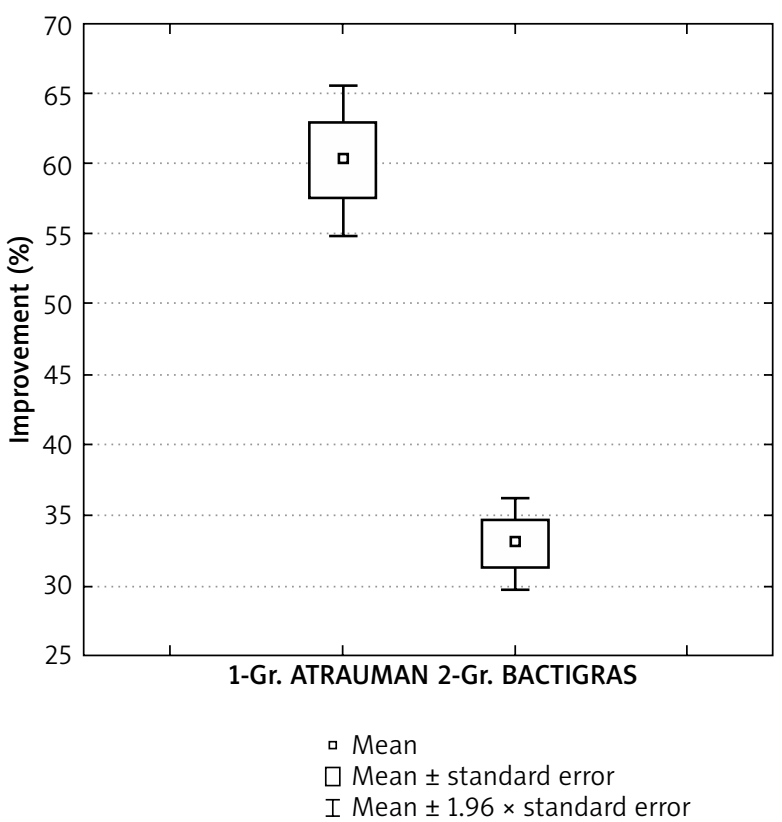

Figure 4. Reduction in the surface wound area in percentage after the treatment in comparison to the output values in both groups of patients (mean value \pm standard error of the mean - SEM)

12]. Nowadays, on the medical market, there are around 3000 different types of dressings used in the healing of chronic wounds: venous leg ulcers, diabetic foot and decubitus ulcers. Nevertheless, the effects of treatment of this kind of wounds with dressings are still not fully satisfactory as they very often do not ensure full recovery of the wound. The great hopes surround dressings which contain active ingredients [13]. The role of active dressing is, among others, to provide optimal environmental conditions for healing, control seepage, provide sufficient moist as well as to protect the skin first and foremost, against mechanical damage (caused by e.g. friction), and secondly against microorganisms, which can get through to the wound and cause infection [14]. In their paper [15], Foster et al. compared the effectiveness of hydrofiber and polymer dressings in a group of 100 patients with acute wounds after surgeries. Despite the fact that the differences in intensity of healing of the wounds between groups of patients treated with two types of dressings were statistically non-significant, the authors, based on the analysis of the therapeutic results obtained, recommend the usage of hydrofiber dressings in order to heal acute wounds [15].

The choice of the most suitable type of active dressing depends on the clinical characteristics of lesions and should be done under a doctor's supervision. In turn, the frequency of changing dressings depends on the severity of seepage. Available specialist dressings for treating pressure ulcers play a very important role in the process of healing the wounds. Even if for the most cases, a unit 
price is higher than a price of traditional dressings, it should be noted that the active dressings can significantly reduce the time of healing, and many of them require changing once every few days. This translates into significant reduction in the total cost of treatment [16]. Similar conclusions are presented in the research conducted by Russel and Carr [17], in which the authors analysed labour costs of people who were changing hydrocolloid dressings during treatment of venous leg ulcers in comparison to the usage of gas-permeable dressings.

In my own research, the therapeutic effectiveness of both types of specialist dressings is very satisfactory. Using these dressings led to a statistically significant decrease in the wound surface area and accelerated the process of healing in both groups of patients, while the therapeutic effect obtained was better for the group using ATRAUMAN Ag dressing containing silver ions. Results of research conducted by other authors indicate that dressings with silver have antibacterial and sterilization properties, they do not create refractoriness and have a low toxicological profile, which may explain their better effectiveness in healing the decubitus ulcers $[6,7,18]$.

Taking into consideration a small number of contraindications to their usage, specialist dressings may be perceived as a valuable supplement to the classic pharmaceutical treatment, which in the future could allow for optimization of therapeutic procedures and improve efficiency of healing the pressure ulcers.

\section{Conclusions}

Employment of a new generation of active specialist dressings enables efficient healing of decubitus, which is manifested by a statistically significant decrease in the surface wound areas. Specialist dressings containing silver ions show greater efficiency in healing the decubitus ulcers in comparison to sterile paraffin gauze. It is justified to continue further, multicentre trials on bigger groups of patients concerning the clinical use of specialist dressings for healing the decubitus, which results will enable optimization of therapeutic procedures and therefore will improve the effectiveness of healing the pressure ulcers.

\section{Conflict of interest}

The authors declare no conflict of interest.

\section{References}

1. Sopata M, Tomaszewska E, Głowacka A. Decubitus - risk evaluation and prophylaxis. Chir Angiol Nurs 2007; 4: 165-9.

2. Zbarańska K. Decubitus - pathogenesis, prophylaxis and treatment. New Medicine 2003; 1: 12-8.

3. Krasowski G, Kruk M. The treatment of decibutus and chronic wounds. Wyd. I. PZWL, Warsaw 2008.

4. Sopata M. Prophylaxia and treatment of decubitus. Puls Medicine 2007; 12: 155.
5. Jawień A, Bartoszewicz M, Przondo-Mordarska A, et al. Guidelines for local and systemic treatment of infected chronic wounds. Wound Treatment 2012; 9: 59-75.

6. Maillard JY, Denyer SP. Discovery of silver. Wound Treatment 2007; 4: 9-12.

7. White RJ. An historical overview on the use of silver in wound management. Br J Nurs 2001; 10: 3-8.

8. Głowacka A. Decubitus - prevention and contemporary preservative treatment methods. Our Affairs 2006; 1: 22-5.

9. Roach R, Dexter C. The practicing physicians' guide to pressure ulcers in 2008. Med Health R I 2009; 92: 30-1.

10. Ramundo J, Pike C, Pittman J. Do prophylactic foam dressings reduce heel pressure injuries? I Wound Ostomy Continence Nurs 2018; 45: 75-82.

11. Jones CM, Rothermel AT, Mackay DR. Evidence-based medicine: wound management. Plast Reconstr Surg 2017; 140: 201-6.

12. Silva DRA, Bezerra SMG, Costa JP, et al. Pressure ulcer dressings in critical patients: a cost analysis. Revista da Escola de Enfermagem da USP 2017; 51: 32-6.

13. Dhivya S, Padma VV, Santhini E. Wounds dressings - a review. J Wound Care 2015; 5: 442-5.

14. Szewczyk MT, Cwajda J, Cierzniakowska K, et al. Chosen treatment aspects of chronic wounds. Medical Guide 2005; 5: 54-60.

15. Foster L, Moore P, Clark S. A comparison of hydrofibre and alginate dressings on open acute surgical wounds. I Wound Care 2000; 9: 442-5.

16. Prior J. The pressure is on: midwives and decubitus ulcers. RCM Midwives J 2002; 5: 196-200.

17. Russel L, Carr J. New hydrofibre and hydrocolloid dressings for chronic wounds. J Wound Care 2000; 9: 169-72.

18. Orsted HL, Rosenthal S, Woodbury MG. Pressure ulcer awareness and prevention program: a quality improvement program through the Canadian Association of Wound Care. J Wound Ostomy Continence Nurs 2009; 36: 178-83. 